06

\title{
Кинетическая модель наноструктурирования аморфной металлической пленки, инициированного миллисекундным тепловым импульсом
}

\author{
(C) Е.Е. Слядников, И.Ю. Турчановский \\ Томский государственный университет систем управления и радиоэлектроники, \\ 634050 Томск, Россия \\ e-mail: eeslyadnikov@gmail.com
}

Поступило в Редакцию 7 августа 2019 г.

В окончательной редакции 20 января 2020 г.

Принято к публикации 3 февраля 2020 г.

\begin{abstract}
Сформулирована модель, описывающая аморфно-нанокристаллическое превращение как при термическом отжиге, так и при импульсном тепловом воздействии миллисекундной длительности. Генерация нанокристаллов по толщине пленки происходит эстафетным образом, т.е. от нагреваемой поверхности движется волна превращения. Предлагаемый подход учитывает характерные особенности импульсного теплового источника: поверхностный характер, кратковременность воздействия, высокую интенсивность. Учитывались малоразмерность, сильная неравновесность системы, термонапряжения, внутренние термические флуктуации, внешний шум от источника энергии.
\end{abstract}

Ключевые слова: аморфно-нанокристаллическое превращение, нанокристаллизация, термонапряжение.

DOI: 10.21883/JTF.2020.07.49448.299-19

\section{Введение}

Аморфные магнитные материалы нашли широкое применение в электротехнике и радиотехнике [1]. Уникальными магнитными свойствами обладают аморфные металлические сплавы с наноструктурой, которая имеет вид беспорядочно ориентированных нанокристаллов в магнитной аморфной матрице. Существуют различные способы получения такого структурного состояния. Первый способ состоит в термическом нагреве аморфного материала [2], в том числе под нагрузкой [3]. Совместные рентгеноструктурные и ЯГР исследования кристаллизации тонкой аморфной пленки сплавов на основе Fe или Со (толщиной 20-30 $\mu \mathrm{m}$, шириной $10 \mathrm{~mm}$, длиной $30 \mathrm{~mm}$ ) при термическом отжиге в течение 30-60 min показали несколько вариантов изменения структуры. В области температур отжига ниже $500^{\circ} \mathrm{C}$ происходит изменение ближнего порядка в аморфном состоянии (структурная релаксация). При температурах отжига от $T_{1} \approx 500^{\circ} \mathrm{C}$ до $T_{2} \approx 700^{\circ} \mathrm{C}$ образуется нанокристаллическая структура со средним размером зерна около $10 \mathrm{~nm}[2,4]$. Если температура отжига превышает $700^{\circ} \mathrm{C}$, то аморфная структура трансформируется в поликристаллическую структуру со средним размером зерна более микрометра.

Для кристаллизации аморфной пленки также используется тепловой импульс миллисекундной длительности (лазером, электронным пучком, электрической лампой, импульсом тока) [4,5]. При однократном облучении пленки мощным световым импульсом с длительностью $0.5 \mathrm{~ms}$ изменение структуры аморфного сплава зависит от его энергии [4]. При плотности мощности им- пульса на единицу объема, превышающей пороговое значение (подводимая к электрической лампе энергия $\left.\varepsilon_{c} \approx 1.1 \mathrm{~kJ}\right)$, формируется поликристаллическая структура со средним размером зерна около микрометра. Если плотность мощности импульса на единицу объема ниже порогового значения, то происходят изменения в его ближнем порядке (структурная релаксация). При облучении серией из $N \approx 20$ световых импульсов с подводимой к лампе энергией меньше пороговой $\varepsilon_{c}$ происходит последовательный рост доли нанокристаллической структуры до насыщения [4]. Механизм такого влияния теплового импульса на нанокристаллизацию аморфного материала остается неясным. По мнению авторов работы [4], возникающая насыщенная нанокристаллическая структура и механизм нанокристаллизации при импульсном тепловом воздействии миллисекундной длительности такие же, что и при термическом отжиге. Однако при термическом отжиге насыщенная нанокристаллическая структура формируется в течение 30-60 min, а при импульсном тепловом воздействии миллисекундной длительности за $10 \mathrm{~ms}$.

Физические условия, в которых протекает нанокристаллизация термическим отжигом и тепловым импульсом, существенно отличаются. Во-первых, при термическом отжиге стационарное распределение температуры по толщине пленки практически однородно, процесс кристаллизации протекает медленно и гомогенно, а нанокристаллы возникают по всему объему термофлуктуационным путем. При импульсном воздействии тепловой источник имеет поверхностный характер, глубина проникновения излучения в материал составляет примерно один микрометр. Дальнейший прогрев пленки на всю 
ее толщину осуществляется теплопроводностью, причем время температурной релаксации (по толщине пленки) имеет один порядок величины с длительностью светового импульса. В условиях поверхностного импульсного нагрева температура поверхности пленки значительно превышает температуру холодной части пленки, и в пленке возникают значительные термонапряжения. Поэтому механизм нанокристаллизации при импульсном тепловом воздействии миллисекундной длительности должен включать, помимо внутренних термических флуктуаций, также атермические флуктуации, инициированные внешним шумом от источника энергии [6].

Во-вторых, образование нанокристаллов в аморфном материале при импульсном воздействии протекает в неизотермическом, неравновесном, нестационарном режиме. Структурная релаксация системы в таких условиях может протекать в бездиффузионном режиме за счет возбуждения динамического ближнего порядка [7], связанного с локализацией электрона и изменением типа химической связи в атомном кластере [8]. Термин „динамический“ означает, что данный ближний порядок возбуждается под действием механических напряжений, амплитуда которых в рассматриваемом случае определяется градиентом температуры. Способ учета этих напряжений за счет добавления вклада энергии упругих деформаций в термодинамический потенциал системы предложен в модели фазового поля, описывающей кристаллизацию металла из расплава [9]. Необходимо развитие этого подхода для описания кинетики аморфнонанокристаллического превращения.

В-третьих, режим распространения аморфно-нанокристаллическом превращения - волновой и связан с атомными процессами теплопроводности. В [10] предложена кинетическая модель, описывающая распространение температурного фронта при термоактивированном аморфно-поликристаллическом превращении в аморфной пленке $\mathrm{Ti}_{50} \mathrm{Cu}_{50}$, инициированном объемным тепловым источником. Показано, что существует температура самопроизвольного поликристаллического превращения $T_{*}$, выше которой система становится неустойчивой относительно малых флуктуаций температуры. Режим распространения превращения - волновой, и связан с атомными процессами теплопроводности, а механизм явления имеет тепловой характер. При тепловом механизме возникновения и распространения фронта превращения тепло, выделившееся при превращении, теплопроводностью передается на соседние участки непревращенного вещества, нагревает их и инициирует самоускоряющийся переход. При температурах ниже $T_{*}$ инициация волнового аморфно-поликристаллического превращения возможна только в случае дополнительного локального нагрева пленки со свободной поверхности.

Целью настоящей работы является формулировка кинетической модели и описание механизма наноструктурирования аморфной металлической пленки при нагреве и термоупругих напряжениях, инициированных поверхностным миллисекундным тепловым импульсом, с учетом внутреннего и внешнего шума.

\section{Модель генерации нанокристаллов одиночным тепловым импульсом}

Рассматривается слой аморфной пленки, в котором протекает процесс нанокристаллизации при умеренной плотности энергии теплового импульса на единицу объема $w_{V}$. Этот режим имеет место при выполнении условия

$$
c \rho\left(T_{1}-T_{0}\right) S l \leq\left(w_{V}+\rho \frac{L}{N}\right) S l \leq c \rho\left(T_{2}-T_{0}\right) S l .
$$

Здесь $S l$ - объем превращающегося слоя, $l$ - толщина превращающегося слоя, $S$ - площадь поверхности одной из граней пленки, $L-$ скрытая удельная теплота аморфно-нанокристаллического превращения, $N$ число импульсов в серии. $T_{1,2}$ - температуры границ нанокристаллизации в режиме отжига, $T_{0}$ - начальная температура слоя, $c$ - теплоемкость среды, $\rho$ - плотность среды. Слой пленки с объемом $S l$ быстро нагревается, возникает градиент температуры $\nabla T \approx \frac{\left[T-T_{0}\right]}{l}$, термонапряжения $\sigma=K \alpha\left[T-T_{0}\right]$, где $K^{-1}-$ коэффициент всестороннего сжатия, $\alpha-$ коэффициент теплового расширения.

Для оценки значений, характеризующих процесс величин, предположим, что тепловой импульс с длительностью $\tau \approx 5 \cdot 10^{-4} \mathrm{~s}$ нагревает пленку с объемом $V=30 \mu \mathrm{m} \times 10 \mathrm{~mm} \times 30 \mathrm{~mm}=9 \cdot 10^{-3} \mathrm{~cm}^{3}$ от температуры $T_{0} \approx 300 \mathrm{~K}$ до температуры $T \approx 900 \mathrm{~K}$. Нагрев начинается с одной из граней поверхности пленки площадью $S=10 \mathrm{~mm} \times 30 \mathrm{~mm}=3 \mathrm{~cm}^{2}$. Тогда энергия теплового импульса равна $\varepsilon(T)=c \rho V\left(T-T_{0}\right) \approx 25 \mathrm{~J}$ $\left(c \approx 0.6 \mathrm{~J} /(\mathrm{g} \cdot \mathrm{K}), \rho \approx 7.7 \mathrm{~g} / \mathrm{cm}^{3}\right)$, мощность теплового импульса равна $\frac{\varepsilon}{\tau} \approx 5 \cdot 10^{4} \mathrm{~J} / \mathrm{s}$. Плотность энергии импульса на единицу объема равна $w_{V}(T)=\frac{\varepsilon T}{V} \approx 2.8 \mathrm{~kJ} / \mathrm{cm}^{3}$, плотность энергии импульса на единицу площади поверхности равна $w_{S}=\frac{\varepsilon}{S} \approx 8.3 \mathrm{~J} / \mathrm{cm}^{2}$. Энергия импульса, приходящаяся на один атом объема, равна $E_{V} \approx 0.75 \mathrm{eV}$, энергия импульса, приходящаяся на один атом поверхности, равна $E_{S} \approx 16 \mathrm{keV}$ (межатомное расстояние равно $\approx 0.35 \mathrm{~nm})$. Плотность мощности импульса на единицу объема равна $\frac{w_{V}}{\tau} \approx 5.6 \cdot 10^{3} \mathrm{~kJ} /\left(\mathrm{s} \cdot \mathrm{cm}^{3}\right)$, плотность мощности импульса на единицу площади поверхности равна $\frac{w_{S}}{\tau} \approx 1.7 \cdot 10^{4} \mathrm{~J} /\left(\mathrm{s} \cdot \mathrm{cm}^{2}\right)$. Поскольку аморфнонанокристаллическое превращение протекает во всем объеме пленки, а не на ее поверхности (сравнение значений $E_{V}$ и $\left.E_{S}\right)$, параметрами энергетического импульса, управляющими процессом превращения, являются величины, приходящиеся на единицу объема. Термические напряжения, инициирующие неупругую необратимую деформацию и определяющие процесс превращения в слое, пропорциональны градиенту температуры в слое. 
Большие значения градиента температуры в слое возможны только в случае, когда длительность теплового импульса меньше времени температурной релаксации по толщине пленки. Поэтому управляющим параметром превращения является плотность мощности импульса на единицу объема $\frac{w_{V}}{\tau}$. Поскольку в нашей задаче $\tau$ меньше или равно времени температурной релаксации, в качестве управляющего параметра можно выбрать $w_{V}$.

Проведем оценку перегрева в превращающемся слое c $l \approx 1 \cdot 10^{-6} \mathrm{~m}$, предполагая, что он нагревается потоком тепла с нагреваемой поверхности $c \rho a \nabla T(a-$ коэффициент температуропроводности) и выделением скрытой теплоты превращения $\rho \frac{L}{N} S l$. Выбирая для простоты линейную зависимость температуры по толщине пленки, получим перегрев за счет потока тепла $\Delta T \approx \frac{600}{30} \approx 20 \mathrm{~K}$, а за счет выделения скрытой теплоты превращения $\Delta T=\frac{L}{N c} \approx 6 \mathrm{~K}(L \approx 74 \mathrm{~J} / \mathrm{g}$, $\left.a \approx 10^{-6} \mathrm{~m}^{2} / \mathrm{s}\right)$. Время температурной релаксации в слое равно $t_{T} \approx \frac{l^{2}}{a} \approx 10^{-6} \mathrm{~s}$. Вследствие различных температур и межатомных расстояний в предыдущем, превращающемся и последующем слоях, в превращающемся слое возникают продольные и сдвиговые напряжения. Для железа $K \approx 3 \cdot 10^{13} \mathrm{~Pa}, \alpha \approx 3 \cdot 10^{-5} \mathrm{~K}^{-1}$, в условиях перегрева слоя $\Delta T \approx(20+6) \mathrm{K}$ величина термонапряжений $\sigma \approx 2 \cdot 10^{10} \mathrm{~Pa}$. Модуль сдвига для железа равен $\mu \approx 7 \cdot 10^{10} \mathrm{~Pa}$, поэтому $\sigma \sim 0.3 \mu$. Находящаяся в этих условиях система становится механически неустойчивой $[11]$.

Дальнейшее рассмотрение основано на кинетической теории Ландау-Халатникова [12]. В этой теории вводится безразмерная величина, характеризующая структурное состояние среды, которая называется параметром порядка $\eta$. В случае наноструктурирования параметром порядка выбираем объемную долю нанокристаллов в единице объема. В аморфном состоянии параметр порядка равен нулю, а в насыщенном нанокристаллическом состоянии параметр порядка равен единице. Процесс структурного превращения рассматривается как временная последовательность структурных состояний среды, описывающаяся изменением параметра порядка со временем, т.е. $\eta=\eta(t)$.

Уравнение Ландау-Халатникова для безразмерного параметра порядка, описывающее структурную релаксацию неравновесной однородной системы к равновесию, имеет вид

$$
\frac{d \eta}{d t}=-t_{\eta}^{-1} \frac{\partial F}{\partial \eta}, \quad \eta(0)=0,
$$

где $t_{\eta}^{-1}=W(T)=v_{0} e^{-\frac{E}{k T}}-$ величина, обратная характерному времени релаксации процесса, пропорциональная частоте колебаний атомов среды $v_{0} \approx 10^{13} 1 / \mathrm{s}$. $E-$ энергия активации, $W(T)$ - скорость превращения, $\eta(0)$ - начальное значение параметра порядка, $F-$ модельный безразмерный неравновесный потенциал.

Воздействие миллисекундного теплового импульса, кроме нагрева системы, переводит ее в деформиро- ванное метастабильное состояние вследствие неисчезающей поперечной жесткости системы. Поэтому модельный потенциал является функцией температуры, деформации $\varepsilon$, параметра порядка, т. е. $F=F(T, \varepsilon, \eta)$. При аморфно-нанокристаллическом превращении первого рода метастабильное аморфное состояние отделено от нанокристаллического состояния энергетическим барьером. Поэтому зависимость $F$ от $\eta$ представляет собой в общем случае модельный асимметричный неравновесный двухямный потенциал.

Отклонение системы от равновесия зависит от изменения температуры и изменения модуля сдвига, и описываться безразмерным параметром неравновесности

$$
\theta=\frac{T-T_{1}}{T_{2}-T_{1}}-6 \frac{\Delta \mu}{\mu_{0}}\left[e_{1}^{2}+e_{2}^{2}+e_{3}^{2}\right],
$$

где $e_{i}-$ безразмерная компонента упругой деформации сдвига $(i=x y, x z, y z=1,2,3) . T_{1}-$ температура равновесия между аморфным и наноструктурным состоянием, $T_{2}$ - температура абсолютной неустойчивости аморфной системы [10], причем $1 \geq \theta \geq 0, \mu_{0}-$ значение модуля сдвига в аморфном состоянии, $\mu_{0}+\Delta \mu-$ значение модуля сдвига в наноструктуре. Например, для сплава $\mathrm{Fe}_{58} \mathrm{Ni}_{25} \mathrm{~B}_{17}$ экспериментально наблюдается явный спад микротвердости по мере увеличения объемной плотности наночастиц [13], поэтому положим $\Delta \mu<0$. При нагревании выше температуры $T_{1}$ и $\tau_{i}=0$ аморфное состояние системы становится метастабильным, неустойчивым относительно конечной термофлуктуации новой структуры с размером зародыша больше критического. При достижении температуры $T_{2}$ аморфное состояние становится абсолютно неустойчивым относительно малой термофлуктуации новой структуры.

Процесс наноструктурирования имеет релаксационный характер, поэтому по мере его протекания модуль сдвига монотонно изменяется до тех пор, пока не обратится в модуль сдвига в нанокристаллической структуре. Модуль сдвига есть результат дальнодействующих корреляций флуктуаций атомной плотности, и может изменяться только в результате взаимной перестройки атомов при структурном переходе. Это требует намного больше времени, чем, например, для смещений атомов, происходящих при распространении упругой волны. Медленность изменения модуля сдвига позволяет исследовать кинетику наноструктурирования кристалла в рамках кинетической теории Ландау-Халатникова. Сдвиговая устойчивость среды, определяемая модулем сдвига, зависит от параметра порядка, поскольку появление макроскопического количества нанокристаллов меняет сдвиговую устойчивость. Поэтому можно предположить, что неравновесный модуль сдвига $\mu$ является функцией неравновесного параметра порядка

$$
\mu=\mu(\eta)=\mu_{0}\left[1+\frac{\Delta \mu}{\mu_{0}} \varphi(\eta)\right], \quad \varphi(\eta)=3 \eta^{2}-2 \eta^{3},
$$

где $\varphi(\eta)$ - безразмерная функция параметра порядка. Из (3) видно, что в аморфном состоянии 
$\varphi(0)=0, \mu(0)=\mu_{0}$, а в наноструктурированном состоянии $\varphi(1)=1, \mu(1)=\mu_{0}+\Delta \mu$.

Для описания деформированного метастабильного состояния среды необходимо к неравновесному размерному термодинамическому потенциалу добавить размерную энергию компонент упругой деформации сдвига

$$
\frac{\mu \varepsilon_{1}^{2}}{2}+\frac{\mu \varepsilon_{2}^{2}}{2}+\frac{\mu \varepsilon_{3}^{2}}{2},
$$

где $\varepsilon_{i}$ - компонента упругой деформации сдвига, $\mu$ неравновесный модуль сдвига. Тогда можно определить безразмерную компоненту упругой деформации сдвига $e_{i}$ :

$$
e_{i}^{2}=\frac{\varepsilon_{i}^{2}}{\varepsilon_{0 i}^{2}}
$$

где $\varepsilon_{0 i}$ - критическая компонента упругой деформация сдвига, которая при температуре $T_{1}$ переводит аморфную систему в абсолютно неустойчивое состояние.

Используя (1)-(5), получим, что в нагретой и деформированной среде разложение безразмерного неравновесного модельного потенциала в окрестности температуры $T_{1}$ по степеням параметра порядка имеет вид

$$
\begin{aligned}
F(\eta)= & \left\{\frac{1}{4} \eta^{2}-\frac{1}{2} \eta^{3}+\frac{1}{4} \eta^{4}+\frac{1}{2}\left[e_{1}^{2}+e_{2}^{2}+e_{3}^{2}\right]\right. \\
& \left.-\frac{1}{12} \theta \varphi(\eta)\right\} .
\end{aligned}
$$

Стационарные однородные решения (1), (6), которые определяются условием

$$
\frac{\partial F(\eta)}{\partial \eta}=\left(\eta-\eta_{1}\right)\left(\eta-\eta_{2}\right)\left(\eta-\eta_{3}\right)=0
$$

равны соответственно

$$
\eta_{1}=0, \quad \eta_{2}(\theta)=\frac{1}{2}[1-\theta], \quad \eta_{3}=1 .
$$

Аморфному состоянию среды соответствует минимум потенциала в точке $\eta_{1}=0$, а насыщенному наноструктурному состоянию - минимум потенциала в $\eta_{3}=1$. Стационарному неустойчивому состоянию соответствует максимум потенциала в $\eta_{2}(\theta)$. Значения изменения потенциала $\Delta F(\eta, \theta)=F(\eta, \theta)-\frac{1}{2}\left[e_{1}^{2}+e_{2}^{2}+e_{3}^{2}\right]$ в стационарных состояниях равны соответственно

$$
\begin{gathered}
\Delta F(0, \theta)=0, \quad \Delta F\left(\frac{1}{2}[1-\theta], \theta\right)=\frac{1}{64}[1-\theta]^{3}\left[1+\frac{\theta}{3}\right], \\
\Delta F(1, \theta)=-\frac{\theta}{12}
\end{gathered}
$$

Величина барьера (водораздела), разделяющего минимумы модельного двухямного потенциала, определяет устойчивость состояния среды и пропорциональна величине

$$
\eta_{2}(\theta)^{3}=\frac{1}{8}[1-\theta]^{3}
$$

которая обращается в нуль при $\theta=1$. При $\theta>0$ аморфное состояние системы становится метастабильным, неустойчивым относительно конечной флуктуации новой структуры с размером зародыша, больше критического. При достижении $\theta=1$ аморфное состояние становится абсолютно неустойчивым относительно малой, но конечной флуктуации новой структуры.

Общее решение уравнения (1) имеет вид

$$
2 t_{\eta}^{-1} t=\ln \frac{\left|\eta-\eta_{2}(\theta)\right|}{|1-\eta||\eta|}-\ln \frac{\left|\eta(0)-\eta_{2}(\theta)\right|}{|1-\eta(0)||\eta(0)|} .
$$

При $\theta>0$ решение (11) разделяет первый квадрант плоскости $(\eta, t)$ на два домена, каждый из которых контролируется одним аттрактором (областью притяжения). Этим аттракторам соответствуют стационарные устойчивые однородные решения (8), а „водоразделу“ между аттракторами - стационарное неустойчивое однородное решение $\eta_{2}(\theta)$.

Безразмерная компонента упругих напряжений в среде определяется как

$$
\sigma_{i}=\frac{\partial F(\eta)}{\partial e_{i}}=\left[1+\frac{\Delta \mu}{\mu_{0}} \varphi(\eta)\right] e_{i} .
$$

При температуре $T<T_{1}$ и $\tau_{i}=0$ устойчивым является состояние с $\eta_{1}=0$. Это означает, что среда находится в аморфном состоянии с модулем сдвига $\mu=\mu_{0}$. По мере роста упругих напряжений уменьшается величина второго корня $\eta_{2}\left(T, e_{i}\right)$, он начинает двигаться влево к корню $\eta_{1}=0$. При температуре $T_{1}<T<T_{2}$ и критическом значении безразмерной упругой сдвиговой деформации

$$
\left[e_{1}^{2}+e_{2}^{2}+e_{3}^{2}\right]_{c}=\left[1-\frac{T-T_{1}}{T_{2}-T_{1}}\right] \frac{\mu_{0}}{6|\Delta \mu|}
$$

метастабильное аморфное состояние $\eta_{1}=0$ становится абсолютно неустойчивым, а поперечная жесткость среды изменяется. Если воздействовать на систему, постепенно повышая сдвиговое напряжение, то при достижении критического значения (13) среда теряет сдвиговую устойчивость, следовательно, возникает неупругая в том числе необратимая деформация.

При наличии в среде необратимой деформации упругая деформация $e_{e l}$ может быть представлена как разность полной деформации е и необратимой деформации $e_{p l}$

$$
e_{e l}=e-e_{p l} .
$$

Если необратимая деформация меняется со временем, то это приводит к дополнительному вязкому напряжению

$$
\sigma_{v}=\frac{\beta}{\mu_{0}} \dot{e}_{p l},
$$

где $\beta-$ коэффициент вязкости. Тогда полное напряжение в среде $\sigma$ равно

$$
\sigma=\sigma_{v}+\sigma_{e l}
$$


Для качественного анализа предположим, что упругая и необратимая деформации в малой окрестности точки перехода связаны линейным релаксационным соотношением Максвелла

$$
\dot{e}_{p l}=\frac{e_{e l}}{t_{\sigma}},
$$

где $t_{\sigma}-$ характерное время релаксации внутренних напряжений. Используя (12), (15), (17), из (16) получим для каждой компоненты полных сдвиговых напряжений

$$
\sigma=\left[1+\frac{\Delta \mu}{\mu_{0}} \varphi(\eta)\right] e_{e l}+\frac{\beta}{\mu_{0} t_{\sigma}} e_{e l} .
$$

Из (18) видно, что при малой упругой сдвиговой деформации $e_{e l} \ll e_{c}$ напряжение подчиняется закону Гука $\sigma=e_{e l}$, а при дальнейшем повышении упругой сдвиговой деформации в точке $e_{c}$ происходит скачкообразное изменение ближнего порядка аморфной среды, генерация нанокристаллов, уменьшение сдвиговых напряжений, вязкое течение среды.

\section{Кинетика неравновесного превращения аморфное состояние-нанокристалли- ческое состояние}

Рассмотрим структурную релаксацию неравновесной неоднородной одномерной (по толщине пленки $l$ ) системы к равновесию

$$
\begin{aligned}
\frac{\partial \eta}{\partial t}= & -t_{\eta}^{-1}\left[\frac{\delta \Delta F}{\delta \eta}-D t_{\eta} \frac{\partial^{2} \eta}{\partial x^{2}}\right] \\
= & -t_{\eta}^{-1}\left[\eta\left(\eta-\eta_{2}(\theta)\right)(\eta-1)-l_{\eta}^{2} \frac{\partial^{2} \eta}{\partial x^{2}}\right], \\
\Delta F(\eta)= & \int d x f[\eta(x)]=\int d x\left[\frac{1}{4} \eta^{2}-\frac{1}{2} \eta^{3}+\frac{1}{4} \eta^{4}\right. \\
& \left.-\frac{1}{12} \theta \varphi(\eta)\right], \\
-\frac{l}{2}<x< & \frac{l}{2}, \eta\left(-\frac{l}{2}, t\right)=1, \eta\left(\frac{l}{2}, t\right)=0, \eta(x, 0)=0 .
\end{aligned}
$$

Здесь $f[\eta(x)]$ - плотность изменения безразмерного неравновесного модельного потенциала, $D$ - коэффициент подвижности параметра порядка, $l_{\eta}=\sqrt{D t_{\eta}}-$ ширина межфазной поверхности, причем $l \gg l_{\eta}$. Граничные условия уравнения (19) выбраны для физического случая начала превращения с левого конца образца. Помимо небольших возмущений устойчивых стационарных состояний, существует еще одна важная группа решений динамического уравнения (19) - автомодельные (подобные) решения. То есть мы будем искать волновое решение в виде

$$
\eta(x, t)=\eta(u), \quad u=x-v t,
$$

где $v$ - константа, скорость, которую необходимо определить. Волну переключения (21) можно представить в виде плоской структуры с профилем, заданным $\eta(u)$, движущимся в трехмерном пространстве со скоростью $v$ по направлению оси $x$. Подставим (21) в (19) и получим обыкновенное дифференциальное уравнение

$$
l_{\eta}^{2} \frac{d^{2} \eta}{d u^{2}}+v t_{\eta} \frac{d \eta}{d u}-\frac{\partial f}{\partial \eta}=0 .
$$

Его решение имеет вид

$$
\begin{gathered}
\eta(u)=\left[1+\exp \left(\frac{u}{l_{\eta}} \operatorname{sign}(\theta)\right)\right]^{-1}, \\
v=\frac{l_{\eta}}{t_{\eta}}\left[1-2 \eta_{2}(\theta)\right]=\frac{l_{\eta}}{t_{\eta}} \theta .
\end{gathered}
$$

Решение (23) выбрано для граничного условия наноструктуры слева, а аморфное состояние - справа. Уравнение (22) определяет величину $\theta$ в качестве „Движущей силы“ для межфазной поверхности и устанавливает связь между скоростью $v$ и параметрами системы $l_{\eta}, t_{\eta}$. Скорость $v$ пропорциональна отклонению системы от равновесия $\theta$, которое измеряется величиной $\left[1-2 \eta_{2}(\theta)\right]$. Из (23) видно, что при температуре $T=T_{1}$ и $e_{i}=0$ (в равновесии) межфазная поверхность не движется, а для $T>T_{1}(\theta>0)$ и $T<T_{1}(\theta<0)$ межфазная поверхность движется в противоположных направлениях, потому что величина $\operatorname{sign}(\theta)$ и скорость $v$ имеют противоположные знаки. Два возможных знака $\operatorname{sign}(\theta)$ проявляют зеркальную симметрию системы: при знаке „,+“ волна движется в направлении увеличения $u$, при знаке ,-“" - обратно.

Рассмотрим рост сферического зародыша, движение межфазной поверхности которого подчиняется трехмерному варианту уравнения (22) с профилем $\eta(u)(23)$. Релаксация системы подчиняется условию диссипации для изменения модельного потенциала трехмерной системы $\Delta F(t)$ (функции Ляпунова)

$$
\begin{aligned}
\frac{d \Delta F(t)}{d t} & =\int d \mathbf{x}\left[\frac{\delta \Delta F}{\delta \eta} \frac{d \eta}{d t}\right]=-t_{\eta}^{-1} \int d \mathbf{x}\left[\frac{\delta \Delta F}{\delta \eta}\right]^{2}<0 \\
\Delta F(t) & =\int d \mathbf{x}\left[f(\eta(\mathbf{x}, t))+\left(\frac{1}{2}\right) l_{\eta}^{2}\left(\frac{d \eta}{d \mathbf{x}}\right)^{2}\right]
\end{aligned}
$$

Для наиболее вероятной флуктуации - сферического зародыша новой структуры радиуса $R$ с характерной шириной межфазной поверхности $l_{\eta}$ в системе с объемом $V$ - условие (24) принимает вид

$$
\begin{aligned}
& \frac{d \Delta F(t)}{d t}=\frac{d \Delta F}{d R} \frac{d R(t)}{d t} \leq 0 \\
\Delta F(R)= & \left\{f(0)\left[V-\frac{4}{3} \pi R^{3}\right]+f(1) \frac{4}{3} \pi R^{3}\right. \\
+ & \left.\frac{l_{\eta}^{2}}{2}\left[\frac{1}{l_{\eta}}\right]^{2} 4 \pi R^{2} l_{\eta}\right\} .
\end{aligned}
$$


Из (25) видно, что критерий роста зародыша $\frac{d R}{d t}>0$ выполняется если $\frac{d \Delta F}{d R}<0$. Из условия $\frac{d \Delta F}{d R}=0$ и (9) можно найти критический радиус зародыша

$$
R_{*}=-l_{\eta}^{2}\left[\frac{1}{l_{\eta}}\right]^{2} l_{\eta} \frac{1}{[f(1, \theta)-f(0, \theta)]}=\frac{12 l_{\eta}}{\theta} .
$$

Рассмотрим эволюцию небольшой флуктуации параметра порядка в трехмерной системе, описываемой трехмерным вариантом уравнения (19)

$$
\Delta \eta(\mathbf{x}, t)=\eta(\mathbf{x}, t)-\eta_{1}=\eta(\mathbf{x}, t)
$$

около стационарного аморфного состояния $\eta_{1}=0$. Разлагая $\frac{\partial f}{\partial \eta}$ в ряд Тейлора около стационарного состояния $\eta_{1}$ с точностью до первого порядка по $\Delta \eta$, из (19) получим

$$
t_{\eta} \frac{\partial \Delta \eta}{\partial t}=-\left[\frac{\partial^{2} f}{\partial \eta^{2}}\left(\eta_{1}\right) \Delta \eta-l_{\eta}^{2} \nabla^{2} \Delta \eta\right] .
$$

Поскольку система имеет конечный объем $V$, разлагая $\Delta \eta(\mathbf{x}, t)$ в ряд Фурье

$$
\begin{gathered}
\Delta \eta(\mathbf{x}, t)=\sum_{\mathbf{k}} \Delta \eta(\mathbf{k}, t) \exp (i \mathbf{k x}), \\
\Delta \eta(\mathbf{k}, t)=\frac{1}{V} \int d \mathbf{x} \Delta \eta(\mathbf{x}, t) \exp (-i \mathbf{k x}),
\end{gathered}
$$

получим из уравнения (28) уравнение для фурьекомпоненты $\Delta \eta(\mathbf{k}, t)$

$$
\frac{\partial \Delta \eta(\mathbf{k}, t)}{\partial t}=\beta(\mathbf{k}) \Delta \eta(\mathbf{k}, t),
$$

$\beta(\mathbf{k})=-t_{\eta}^{-1}\left[l_{\eta}^{2} k^{2}+\frac{\partial^{2} f}{\partial \eta^{2}}\left(\eta_{1}\right)\right]=-t_{\eta}^{-1}\left[l_{\eta}^{2} k^{2}+\frac{1}{2}(1-\theta)\right]$.

Решение (30) имеет вид

$$
\begin{gathered}
\Delta \eta(\mathbf{k}, t)=\Delta \eta(\mathbf{k}, 0) \exp (\beta(\mathbf{k}) t), \\
\Delta \eta(\mathbf{k}, 0)=\frac{1}{V} \int d \mathbf{x} \Delta \eta(\mathbf{x}, 0) \exp (-i \mathbf{k x}),
\end{gathered}
$$

где коэффициент $\Delta \eta(\mathbf{k}, 0)$ определяется из начального условия $\Delta \eta(\mathbf{x}, 0)$. Как в случае однородных флуктуаций, коэффициент усиления $\beta$ определяет направление и скорость эволюции, так называемый временной масштаб эволюции $\tau(\mathbf{k})=|\beta(\mathbf{k})|^{-1}$. Если $\frac{\partial^{2} f}{\partial \eta^{2}}\left(\eta_{1}\right)>0$ (аморфное состояние $\eta_{1}$ стабильно), то $\beta(\mathbf{k})<0$ для всех волновых векторов. Если $\frac{\partial^{2} f}{\partial \eta^{2}}\left(\eta_{1}\right)<0$ (аморфное состояние $\eta_{1}$ нестабильно), то $\beta(\mathbf{k})<0$ для волновых векторов $k>k_{n}$, а $\beta(\mathbf{k})>0$ для волновых векторов $0<k<k_{n}$. И $\beta(\mathbf{k})=0$ для волновых векторов с нейтральным волновым вектором $k=k_{n}$

$$
k_{n}^{2}=-\frac{1}{l_{\eta}^{2}} \frac{\partial^{2} f}{\partial \eta^{2}}\left(\eta_{1}\right) .
$$

Если

$$
\frac{\partial^{2} f}{\partial \eta^{2}}\left(\eta_{1}\right)=\left[\frac{1}{2}(1-\theta)\right]_{\theta \rightarrow 1} \rightarrow 0,
$$

то аморфное состояние $\eta_{1}$ теряет устойчивость по отношению к однородным флуктуациям с $k \rightarrow 0$ и распадается. Сравнивая анализ динамической стабильности с анализом термодинамической устойчивости, можно увидеть, что устойчивость состояния определяется знаком $\frac{\partial^{2} f}{\partial \eta^{2}}\left(\eta_{1}\right)$, а скорость релаксации системы к аморфному состоянию равна

$$
\beta(\mathbf{k})=-t_{\eta}^{-1}\left[l_{\eta}^{2} k^{2}+\frac{\partial^{2} f}{\partial \eta^{2}}\left(\eta_{1}\right)\right]=-t_{\eta}^{-1}\left[l_{\eta}^{2} k^{2}+12(1-\theta)\right] .
$$

Видно, что при $\theta \rightarrow 1, k \rightarrow 0$ скорость релаксации $\beta \mathbf{k}$, $\theta \rightarrow 0$. Отметим, что флуктуации параметра порядка должны быть не только малыми по амплитуде, но и не должны иметь слишком большое волновое число, так что $k \leq l_{\eta}^{-1} \leq \frac{1}{a}$, где $a-$ межатомное расстояние.

Рассмотрим стохастическую структурную релаксацию неравновесной неоднородной одномерной (по толщине пленки $l$ ) системы к равновесию, описываемую уравнением Ланжевена [6]

$$
\begin{gathered}
\frac{\partial \eta}{\partial t}=-t_{\eta}^{-1}\left[\eta\left(\eta-\eta_{2}(\theta)\right)(\eta-1)-l_{\eta}^{2} \frac{\partial^{2} \eta}{\partial x^{2}}-\xi\right], \\
P(\xi)=\frac{1}{\sqrt{2 \pi} \sigma} \exp \left(-\frac{\xi^{2}}{2 \sigma^{2}}\right), \quad\langle\xi(x, t)\rangle=0, \\
\langle\xi(x, t) \xi(0,0)\rangle=\sigma^{2} \delta(x) \delta(t) .
\end{gathered}
$$

Согласно Ланжевену, безразмерная случайная переменная $\xi$ имеет нормальное гауссовское распределение $P(\xi)$ с нулевым средним, а автокорреляционная функция является дельта-функцией Дирака (белый шум), угловые скобки означают усреднение по времени и координате. Наличие дельта-функций Дирака означает, что конечные корреляции флуктуирующей силы существуют на временном и пространственном интервале, много меньшем, чем время релаксации $t_{\eta}$ и ширина межфазной поверхности $l_{\eta}$. Случайная сила $\xi$ является суммой двух некоррелированных случайных сил $\xi_{1}$ и $\xi_{2}$ различной физической природы. Силы $\xi_{1}$ и $\xi_{2}$ имеют нормальные гауссовские распределения $P\left(\xi_{1}\right)$ и $P\left(\xi_{2}\right)$, нулевые средние и дисперсии $\sigma_{1}^{2}=\frac{k T}{\Gamma}, \sigma_{2}^{2}=\theta(T) \frac{E_{V}}{\Gamma}$. Дисперсия (интенсивность) силы $\xi$ равна $\sigma^{2}=\sigma_{1}^{2}+\sigma_{2}^{2}=\frac{k T}{\Gamma}+\theta(T) \frac{E_{V}}{\Gamma}$ и состоит из двух членов. Первый член $\frac{k T}{\Gamma}$, пропорциональный температуре $T$, связан с внутренними термическими флуктуациями. Второй член $\theta(T) \frac{E_{V}}{\Gamma}$, пропорциональный энергии импульса, приходящейся на один атом объема, связан с внешним шумом от источника энергии. Здесь $k-$ постоянная Больцмана, $\Gamma-$ нормировочный множитель, имеющий размерность энергии в расчете на один атом и величину порядка энергии активации процесса релаксации $E \approx 1 E v \cdot \theta(T) \equiv\left(w_{V}(T)-w_{V}\left(T_{1}\right)\right)-$ 
тета-функция, которая равна нулю, если энергия импульса $\varepsilon=c \rho V\left(T-T_{0}\right)$ нагревает пленку ниже температуры $T_{1}$, и равна единице, если энергия импульса $\varepsilon=c \rho V\left(T-T_{0}\right)$ нагревает пленку выше температуры $T_{1}$.

Вычислим изменение модельного потенциала системы с объемом $V$ в аморфном состоянии $\eta_{1}=0$, принимая во внимание как внутренние термические флуктуации [12], так и случайный шум от внешнего источника энергии [6]. По Больцману вероятность неравновесного состояния пропорциональна фактору

$$
\exp \left(-\frac{\Gamma \Delta F}{k T+\theta(T) E_{V}}\right)
$$

где $\Delta F-$ флуктуационая часть изменения модельного потенциала системы. Различные состояния системы описываются различными конкретными распределениями параметра порядка $\Delta \eta(\mathbf{x})=\eta(\mathbf{x})-\eta_{1}=\eta(\mathbf{x})$. Распределение вероятностей неравновесных состояний имеет вид

$$
\begin{gathered}
P[\eta(\mathbf{x})] \sim \exp \left(-\frac{\Gamma \Delta F[\eta(\mathbf{x})]}{2\left(k T+\theta(T) E_{V}\right)}\right), \\
\Delta F[\eta(\mathbf{x})]=\int d \mathbf{x}\left[\frac{\partial^{2} f}{\partial \eta^{2}}\left(\eta_{1}\right)(\Delta \eta)^{2}+l_{\eta}^{2}\left(\frac{\partial \Delta \eta}{\partial \mathbf{x}}\right)^{2}\right] \\
=V \sum_{\mathbf{k}}\left[\frac{\partial^{2} f}{\partial \eta^{2}}\left(\eta_{1}\right)+l_{\eta}^{2} k^{2}\right]|\Delta \eta(\mathbf{k})|^{2},
\end{gathered}
$$

где $\Delta F-$ флуктуационная часть изменения модельного потенциала, учитывающая только первый порядок по $(\Delta \eta)^{2}$. Усреднение по статистическому ансамблю (35), (36) эквивалентно усреднению по времени для уравнения Ланжевена (33), (34). Видно, что $\Delta F$ диагонализована в фурье-пространстве и представляет собой сумму членов, зависящих только от одного волнового вектора k. Коэффициенты перед этими членами пропорциональны коэффициенту усиления $\beta(\mathbf{k})$. Из (35), (36) видно, что распределение вероятностей состояний представляет собой произведение распределений Гаусса, каждое из которых зависит от волновых векторов $\mathbf{k}$ и - $\mathbf{k}$. Следовательно, индивидуальные моды $\Delta \eta(\mathbf{k})$ статистически независимы, и эволюцию мод $\mathbf{c} \mathbf{k}$ и $\mathbf{k}^{\prime} \neq-\mathbf{k}$ можно рассматривать независимо. Вычисляя среднеквадратическую флуктуацию параметра порядка по распределению (35), (36), получим

$$
\left\langle|\Delta \eta(\mathbf{k})|^{2}\right\rangle=\frac{k T+\theta(T) E_{V}}{V \Gamma}\left[\frac{\partial^{2} f}{\partial \eta^{2}}\left(\eta_{1}\right)+l_{\eta}^{2} k^{2}\right]^{-1} .
$$

Выражение (37) может быть использовано при

$$
\frac{\partial^{2} f}{\partial \eta^{2}}\left(\eta_{1}\right)>0
$$

поскольку в обратном случае получается отрицательное выражение среднего квадрата флуктуаций для длинноволновых мод $k \rightarrow 0$. Это значит, что выражение (37) применимо только для условно устойчивого, метастабильного состояния $\eta_{1}$. Вычислим средний квадрат флуктуаций в одной точке координатного пространства

$$
\left\langle\Delta \eta(0)^{2}\right\rangle=\left\langle|\Delta \eta(0)|^{2}\right\rangle=\frac{k T+\theta(T) E_{V}}{V \Gamma}\left[\frac{\partial^{2} f}{\partial \eta^{2}}\left(\eta_{1}\right)\right]^{-1}
$$

Выражение (38) показывает, что интенсивность флуктуаций параметра порядка обратно пропорционально величине $\frac{\partial^{2} f}{\partial \eta^{2}}\left(\eta_{1}\right)$. Хотя при обычных условиях $\frac{\partial^{2} f}{\partial \eta^{2}}\left(\eta_{1}, \theta=0\right)=\frac{1}{2} \quad$ флуктуации (37), (38) малы, существуют необычные неравновесные условия $\frac{\partial^{2} f}{\partial \eta^{2}}\left(\eta_{1}, \theta>0\right)=\frac{1}{2}[1-\theta]$, когда флуктуации становятся большими. Эти условия выполняются, когда $V \rightarrow 0$ или когда $\frac{\partial^{2} f}{\partial \eta^{2}}\left(\eta_{1}, \theta\right) \rightarrow 0$, при $\theta \rightarrow 1$ и $k \rightarrow 0$, т. е. система приближается к критической точке, и усиливаются длинноволновые флуктуации. Из (38) видно, что при малой плотности энергии импульса $(\theta(T)=0)$ главную роль играют внутренние термические флуктуации, а при большой плотности энергии импульса $\left(E_{V} \gg k T\right)-$ флуктуации, инициированные внешним шумом от источника энергии.

Выражение (37) можно использовать для вычисления двухточечной пространственной корреляционной функции флуктуаций параметра порядка

$$
\begin{gathered}
K(\mathbf{r})=\langle\Delta \eta(\mathbf{r}) \Delta \eta(0)\rangle=\sum_{\mathbf{k}} \exp (i \mathbf{k r})\left\langle|\Delta \eta(\mathbf{k})|^{2}\right\rangle \\
\sim \frac{1}{4 \pi|\mathbf{r}|} \exp \left(-\frac{|r|}{R_{c}}\right) \\
R_{c}=\frac{l_{\eta}}{\sqrt{\frac{\partial^{2} f}{\partial \eta^{2}}\left(\eta_{1}, \theta\right)}}=\frac{l_{\eta}}{\sqrt{\frac{1}{2}[1-\theta]}}
\end{gathered}
$$

где фундаментальная величина $R_{c}$, называемая корреляционным радиусом флуктуаций, определяет масштаб длины, на которой корреляция флуктуаций заметно уменьшается, и характеризует ближний порядок системы. Если достигается критическая точка $\theta \rightarrow 1$, то $R_{c} \rightarrow \infty$ и $K(\mathbf{r}) \sim \frac{1}{4 \pi|\mathbf{r}|}$. Это означает, что флуктуации параметра порядка коррелируют на очень больших расстояниях. При $|\mathbf{r}| \rightarrow 0 K(\mathbf{r}) \rightarrow \infty$, и это означает, что выражение (39) неприменимо при $l_{\eta}^{2} k^{2}<1, k<\frac{1}{l_{\eta}} \leq \frac{1}{a}$. То есть коротковолновые флуктуации запрещены из-за атомной природы вещества.

Из (26), (40) видно, что при увеличении неравновесности радиус корреляции флуктуаций параметра порядка увеличивается, а радиус критического зародыша уменьшается. Поэтому сушествует критическое значение $\theta_{*}$, при котором эти величины совпадут

$$
\frac{l_{\eta}}{\sqrt{\frac{1}{2}\left[1-\theta_{*}\right]}}=\frac{12 l_{\eta}}{\theta_{*}}, \quad \theta_{*} \approx 1-\frac{2}{(12)^{2}} .
$$


Из (41) видно, что при достижении критического значения $\theta_{*}$ из флуктуаций параметра порядка происходит генерация критических зародышей новой структуры с радиусом $R_{*}=\frac{12 l_{\eta}}{\theta_{*}} \approx R_{\min }$, где $R_{\min }=12 l_{\eta}-$ минимальный радиус критического зародыша. Если для простоты положить ширину межфазной поверхности, равной трем межатомным расстояниям $\left(l_{\eta}=3 a\right)$, то радиус критического зародыша составит $R_{\min } \approx 12 \mathrm{~nm}$, что превышает размеры нанокристаллов, обнаруженных экспериментально [4].

При воздействии теплового импульса в слое увеличивается радиус корреляции флуктуаций параметра порядка, изменяется ближний порядок аморфной среды, генерируются нанокристаллы, внутренняя энергия и упругая энергия превращенного слоя системы понижаются, выделяется скрытая теплота превращения $L / N$, релаксируют напряжения. Эта выделенная теплота превращения, наряду с пришедшим теплом от поверхностного теплового источника, нагревает следующий слой материала, в котором возникают термонапряжения, что вместе вызывает генерацию нанокристаллов уже в этом слое. Слои, в которых нанокристаллизация завершилась, просто являются проводниками тепла от поверхностного теплового источника к слою, в котором протекает превращение. Так, эстафетным образом происходит генерация нанокристаллов по всей толщине пленки, т. е. от нагреваемой поверхности движется волна превращения.

\section{Обсуждение результатов}

В неупорядоченных системах, например в аморфных металлических сплавах, с относительно легко изменяющими внутренними параметрами, могут возникать локализованные состояния электронов [8]. Допустим, что в аморфной металлической пленке возникла флуктуация параметра порядка с радиусом, меньшим радиуса критического зародыша. В отсутствие коллективизированных электронов такая флуктуация приводит к увеличению термодинамического потенциала системы и через некоторое время, очевидно, она „распадется“. Однако при подходящем знаке изменения $\eta$ (при образовании зародыша кристаллической фазы) она приводит к понижению потенциальной энергии электрона. Если возникающая при образовании зародыша кристаллической фазы потенциальная яма оказывается достаточно глубокой и широкой, то электрон локализуется в ней. При локализации энергия электрона понижается, причем в некоторых условиях это понижение может оказаться большим, чем увеличение термодинамического потенциала, связанного с флуктуацией. Тогда возникновение флуктуации, вблизи которой локализован электрон, будет термодинамически выгодным, изменение $\eta$ станет стационарным и не будет „распадаться“. При определенных значениях параметров радиус электронного состояния оказывается достаточно большим, и область локализации электрона захватывает большое число атомов.

Из полученных выше теоретических результатов вытекает следующая физическая картина наноструктурирования при различных режимах теплового воздействия. При термическом отжиге стационарное распределение температуры по толщине пленки практически однородно, термонапряжения отсутствуют, параметр неравновесности мал. Процесс превращения протекает медленно и гомогенно, а зародыши новой фазы медленно возникают по всему объему термофлуктуационным путем, так как $w_{V}=0$ и фактор $\exp \left(-\frac{\Gamma \Delta F}{k T}\right)$. Если радиус флуктуации параметра порядка превышает характерную толщину межфазной поверхности $l_{\eta} \approx 1 \mathrm{~nm}$, то глубина периодического потенциала в области флуктуации становится большой. Электрон локализуется в области флуктуации параметра порядка [8], стабилизирует эту область ближнего порядка и образует новую химическую связь. Так возникает нанокристалл с радиусом $R_{c}$ меньше критического $R_{\min }$. Скорость образования и роста критических зародышей термофлуктуационным путем экспоненциально мала, поэтому при температуpe $T_{1}<T<T_{2}$ реализуется наноструктурное состояние. При температурах выше $T_{2}$, скорость образования и роста критических зародышей термофлуктуационным путем велика, поэтому реализуется поликристаллическое состояние.

В режиме импульсного теплового воздействия распределение температуры по толщине пленки неоднородно, возникают термонапряжения, параметр неравновесности велик $\theta \leq \theta_{*}$. Флуктуации параметра порядка с радиусом $R_{c}$ возникают быстро вследствие содействия как внутренних термических флуктуаций, так и атермических флуктуаций, инициированных внешним шумом от источника энергии. Если плотность энергии одиночного импульса достигает порогового значения $w_{V}\left(T_{2}\right)$, то температура пленки становится равной $T_{2}$, а параметр неравновесности достигает критического значения $\theta=\theta_{*}$. Радиус корреляции флуктуаций параметра порядка (радиус ближнего порядка) $R_{c}$ становится равным радиусу критического зародыша $R_{\min }$, быстро генерируются критические зародыши (фактор $\left.\exp \left(-\frac{\Gamma \Delta F}{k T+\theta(T) E_{V}}\right)\right)-$ нанокристаллы с радиусом $R_{\min }$. Скорость превращения $W(T)$ становится большой и соответственно большой плотность мощности тепловыделения $Q=\rho L W(T)$ за счет превращения. Плотности мощности теплового источника $\frac{w_{V}}{\tau}$ достаточно, чтобы инициировать поликристаллическое превращение с поверхности пленки и на время действия импульса нагреть пленку выше $T_{2}$. Плотности мощности тепловыделения $Q$ за счет превращения достаточно, чтобы самостоятельно поддерживать распространение волны превращения. При температуре выше $T_{2}$ скорость роста критических зародышей велика, превращение протекает полностью, и реализуется поликристаллическое состояние. Поэтому при одиночном 
мощном импульсе формируется поликристаллическая структура.

При энергии одиночного импульса ниже $w_{V}\left(T_{2}\right)$ температура пленки $T_{1}<T<T_{2}$, термические напряжения в области превращения велики, но параметр неравновесности не достигает критического значения $\theta<\theta_{*}$. Вследствие содействия как внутренних термических флуктуаций, так и атермических флуктуаций, инициированных внешним шумом от источника энергии, быстро (фактор $\left.\exp \left(-\frac{\Gamma \Delta F}{k T+\theta(T) E_{V}}\right)\right)$ возникают области ближнего порядка с радиусом корреляции флуктуаций, меньшим радиуса критического зародыша $R_{c}<R_{\min }$. Если радиус флуктуации параметра порядка превышает $l_{\eta} \approx 1 \mathrm{~nm}$, то электрон локализуется в области флуктуации параметра порядка, стабилизирует эту область ближнего порядка и образует новую химическую связь. Так возникает нанокристалл с радиусом $R_{c}$ меньше критического $R_{\min }$. Скорость превращения $W(T)$ небольшая и соответственно небольшая плотность мощности тепловыделения $Q / N$ за счет генерации нанокристаллов с $R_{c}<R_{\min }$. Значения величин $w_{V} / \tau$ и $Q / N$ достаточны, чтобы генерировать нанокристаллы с поверхности и поддерживать распространение волны генерации нанокристаллов в пленке с плотностью, далекой от насыщения. Так, эстафетным образом происходит генерация нанокристаллов по всей толщине пленки, т. е. от нагреваемой поверхности движется волна превращения. Скорость образования и роста критических зародышей термофлуктуационным путем экспоненциально мала, поэтому при температуpe $T_{1}<T<T_{2}$ реализуется наноструктурное состояние. Для серии импульсов каждый последующий импульс инициирует дополнительную генерацию нанокристаллов в пленке вплоть до насыщения, так, возникает нанокристаллическая структура.

\section{Заключение}

Таким образом, механизм нанокристаллизации при импульсном тепловом воздействии миллисекундной длительности должен включать, помимо внутренних термических флуктуаций, также атермические флуктуации, инициированные внешним источником энергии. Генерация нанокристаллов с радиусом меньше критического происходит эстафетным образом по толщине пленки, т.е. от нагреваемой поверхности движется волна превращения. Для серии импульсов каждый последующий импульс инициирует дополнительную генерацию нанокристаллов в пленке вплоть до насыщения, так, возникает нанокристаллическая структура. Предложенная модель описывает физическую картину аморфнонанокристаллического превращения как при термическом отжиге, так и при импульсном тепловом воздействии миллисекундной длительности.

\section{Финансирование работы}

Работа выполнена в рамках государственного задания ИФПМ СО РАН, проект III.23.2.8.

\section{Конфликт интересов}

Автор заявляет, что конфликт интересов отсутствует.

\section{Список литературы}

[1] McHenry M.E., Willard M.A., Laughlin D.E. // Progr. Mater. Sci. 1999. Vol. 44. N 4. P.291-433.

[2] Yoshizawa Y., Oguma S., Yamauchi K. // J. Appl. Phys. 1988. Vol. 64. N 10. P. 6044-6046.

[3] Пермякова И.Е., Глезер А.М., Савченко Е.С., Щетинин И.В. // Известия РАН. Сер. физ. 2017. Т. 81. № 11. C. $1458-1465$.

[4] Назипов Р.А., Митин А.В., Выжсимов Ю.М., Зюзин Н.А., Пятаев А.В. // Физика и химия обработки материалов. 2013. № 6. C. 25-31.

[5] Sypień A., Kusinski J., Stobiecki T., Czapkiewicz M. // Solid State Phenomena. 2003. Vol. 94. P. 75-78.

[6] Хорстхемке В., Лефевр Р. Индуцированные шумом переходы: теория и применение в физике, химии и биологии. М.: Мир, 1987. 400 с.

[7] Егорушкин В.Е., Мельникова Н.В. // ЖЭТФ. 1993. Т. 103. Вып. 2. C. 214-226.

[8] Кривоглаз М.А. // УФН. 1973. Т. 111. Вып. 4. С. 617-654.

[9] Slutsker J., Thornton K., Roytburd A.L., Warren J.A., McFadden G.B. // Phys. Rev. 2006. Vol. 74. P. 014103-112.

[10] Слядников Е.Е., Хон Ю.А., Каминский П.П., Турчановский И.Ю. // ЖТФ. 2018. Т. 88. Вып. 3. С. 374-379.

[11] Борн М., Кунь X. Динамическая теория кристаллических решеток. М.: ИЛ, 1958. 488 с.

[12] Ландау Л.Д., Лифиши Е.М. Физическая кинетика. М.: Наука, 2001. 528 с.

[13] Глезер А.М., Манаенков С.Е., Пермякова И.Е., Попкова Н.А. // Вестник ТГУ. 2010. Т. 15. Вып. 3. С. 1169-1176. 